\title{
Approaching the matched filter bound with block transmission techniques
}

\author{
Fábio Silva ${ }^{1,4 *}$, Rui Dinis ${ }^{1,2}$, Nuno Souto ${ }^{1,3}$ and Paulo Montezuma ${ }^{2,4}$ \\ 1 Instituto de Telecomunicações, Lisboa, Portugal \\ 2 DEE, FCT-UNL, Portugal \\ 3 ISCTE, Lisboa, Portugal \\ 4 UNINOVA, Caparica, Portugal
}

\begin{abstract}
Block transmission techniques, with appropriate cyclic prefixes and employing frequency-domain equalisation techniques, have been shown to be suitable for high data rate transmission over severely time-dispersive channels. The most popular techniques based on this concept are orthogonal frequency division multiplexing (OFDM) and single-carrier with frequency-domain equalisation (SC-FDE).

In this paper, we consider OFDM and SC-FDE transmission schemes and study the impact of the number of multipath components and the diversity order on their performance. It is shown that when we have a high number of separable multipath components, the asymptotic performance of both schemes approaches the matched filter bound, even without diversity. When we have diversity, the performance approaches the matched filter bound faster, with a small number of separable multipath components. It was also observed that the SC-FDE has an overall performance advantage over the OFDM option, especially when employing the iterative block decision feedback equaliser with turbo equalisation and for high code rates. Copyright (C) 2011 John Wiley \& Sons, Ltd.
\end{abstract}

\section{KEY WORDS}

matched filter bound; SC-FDE; OFDM; IB-DFE: Iterative Block Decision Feedback Equaliser; turbo equalisation; diversity

*Correspondence

Fábio Silva, Instituto de Telecomunicações, Lisboa, Portugal.

E-mail: fsilva@|x.it.pt

Received 2 May 2011; Revised 13 August 2011; Accepted 17 August 2011

\section{INTRODUCTION}

It is widely recognised that bock transmissions combined with frequency-domain detection schemes are suitable for broadband wireless systems. Among these, the most popular schemes are orthogonal frequency division multiplexing (OFDM) modulations [1] and single-carrier with frequency-domain equalisation (SC-FDE) modulations [2]. In both cases, we employ frequency-domain equalisation (FDE) at the receiver, whose implementation can be very efficient because the discrete Fourier transform (DFT)/inverse DFT operations can be realised using the fast Fourier transform algorithm. Moreover, the receiver complexity is almost independent of the channel impulse response, making them suitable for severely timedispersive channels $[3,4]$. Because of the lower envelope fluctuations of the transmitted signals, SC-FDE schemes are especially appropriate for the uplink transmission (i.e. the transmission from the mobile terminal to the base station), and OFDM schemes are preferable for the downlink transmission (i.e. the transmission from the base station to the mobile terminal) because of lower signal processing requirements at the receivers $[3,4]$.

The conventional receiver for SC-FDE schemes is a linear FDE. However, it is known that nonlinear equalisers outperform linear equalisers [5]. Iterative block decision feedback equaliser (IB-DFE: Iterative Block Decision Feedback Equaliser) [6] is a promising iterative FDE technique for SC-FDE that was first proposed in [7] and extended to diversity scenarios [8] and layered space-time schemes [9]. These receivers can be regarded as lowcomplexity turbo FDE schemes [10,11], where the channel decoder is not involved in the feedback. True turbo FDE schemes can also be designed based on the IBDFE: Iterative Block Decision Feedback Equaliser concept $[12,13]$. It was observed that the asymptotic performance 
of IB-DFE: Iterative Block Decision Feedback Equaliser schemes can be sometimes very close to the matched filter bound (MFB), but in other cases, it is relatively far from it [8]. However, it is not clear under which circumstances we can expect performances close to the MFB.

In this paper, we study the impact of the number of multipath components and the diversity order in the asymptotic performance of SC-FDE and OFDM schemes and investigate under which conditions the asymptotic can be close to the MFB. This paper is organised as follows: OFDM and SC-FDE receivers are described in Section 2. Analytical expressions for the MFB, when we have multipath propagation and diversity, are presented in Section 3. A set of performance results is presented in Section 4, and Section 5 is concerned with the conclusions of this paper.

\section{SYSTEM DESCRIPTION}

In this paper, we consider wireless systems employing OFDM and SC-FDE block transmission techniques. In a conventional OFDM scheme, the time-domain block is $\left\{s_{n} ; n=0,1, \ldots, N-1\right\}=\operatorname{IDFT}\left\{S_{k} ; k=0,1, \ldots, N-1\right\}$, with $S_{k}$ denoting the frequency-domain data symbols to be transmitted, associated to the $k$ th subcarrier, which are selected from a given constellation (e.g. a quadrature phase-shift keying (QPSK) constellation). On the other hand, for a SC-FDE scheme, the time-domain symbols $\left\{s_{n} ; n=0,1, \ldots, N-1\right\}$ are directly selected from the constellation. For both block transmission schemes, a cyclic prefix, with length longer than the overall channel impulse response, is appended leading to the signal $\left\{s_{n}^{C P} ; n=-N_{G}, \ldots, N-1\right\}$, which is transmitted over a time-dispersive channel.

The transmission structures for both OFDM and SCFDE schemes are depicted in Figures 1(a) and 1(b), respectively.

We consider receivers with $N_{R x}$ diversity branches for both schemes. The signal associated to the $l$ th branch is sampled, and the cyclic prefix is removed leading to the time-domain block $\left\{y_{n}^{(l)} ; n=0,1, \ldots, N-1\right\}$. The corresponding frequency-domain block, obtained after an appropriate size- $N$ DFT operation, is $\left\{Y_{k}^{(l)} ; k=0,1, \ldots\right.$, $N-1\}$, where $Y_{k}^{(l)}$ can be written as

$$
Y_{k}^{(l)}=S_{k} H_{k}^{(l)}+N_{k}^{(l)}
$$

with $H_{k}^{(l)}$ denoting the overall channel frequency response between the transmit antenna and the $l$ th receive antenna for the $k$ th frequency, $S_{k}$ denoting the frequency-domain of the transmitted block and $N_{k}^{(l)}$ denoting the corresponding channel noise.

\subsection{Orthogonal frequency division multiplexing receiver structure}

Figure 2 presents a maximal-ratio combining [14] diversity scheme, which is implemented for each subcarrier $k$. The received sample for the $l$ th receive antenna and the $k$ th subcarrier is given by Equation (1). The analog-to-digital conversion, serial/parallel conversion and demodulation operations are implicit in the block $R x$.

The equalised samples $\left\{\tilde{S}_{k} ; k=0,1, \ldots, N-1\right\}$ are

$$
\tilde{S}_{k}=\sum_{l=1}^{N_{R x}} F_{k}^{(l)} Y_{k}^{(l)}
$$

where $\left\{F_{k}^{(l)} ; k=0,1, \ldots, N-1\right\}$ is the set of FDE coefficients related to the $l$ th diversity branch, denoted by

$$
F_{k}^{(l)}=\frac{H_{k}^{(l) *}}{\sum_{l^{\prime}=1}^{N_{R x}}\left|H_{k}^{\left(l^{\prime}\right)}\right|^{2}}
$$

Applying Equations (1) and (3) to Equation (2), the corresponding equalised samples can then be given by

$$
\tilde{S}_{k}=S_{k}+\frac{\sum_{l=1}^{N_{R x}} H_{k}^{(l) *}}{\sum_{l^{\prime}=1}^{N_{R x}}\left|H_{k}^{\left(l^{\prime}\right)}\right|^{2}} N_{k}^{(l)}
$$

\subsection{Iterative block decision feedback equaliser receiver structure}

Although a linear FDE leads to good performance for OFDM schemes, the performance of SC-FDE can be improved if the linear FDE is replaced by an IB-DFE: Iterative Block Decision Feedback Equaliser [7]. The receiver structure is depicted in Figure $3[6,8]$. The analog-to-digital conversion, the serial/parallel conversion and the demodulation operation are implicit in the block $R x$.

In this case, for the $i$ th iteration, the frequency-domain block at the output of the equaliser is $\left\{\tilde{S}_{k}^{(i)} ; k=\right.$ $0,1, \ldots, N-1\}$, with

$$
\tilde{S}_{k}^{(i)}=\sum_{l=1}^{N_{R x}} F_{k}^{(l, i)} Y_{k}^{(l)}-B_{k}^{(i)} \hat{S}_{k}^{(i-1)}
$$

where $\left\{F_{k}^{(i)} ; k=0,1, \ldots, N-1\right\}$ are the feedforward coefficients and $\left\{B_{k}^{(i)} ; k=0,1, \ldots, N-1\right\}$ are the feedback coefficients. $\left\{\hat{S}_{k}^{(i-1)} ; k=0,1, \ldots, N-1\right\}$ is the DFT of the block $\left\{\hat{s}_{n}^{(i-1)} ; n=0,1, \ldots, N-1\right\}$, with $\hat{s}_{n}$ denoting the 'hard decision' of $s_{n}$ from the previous FDE iteration. It can be shown that the optimum coefficients $B_{k}$ and 


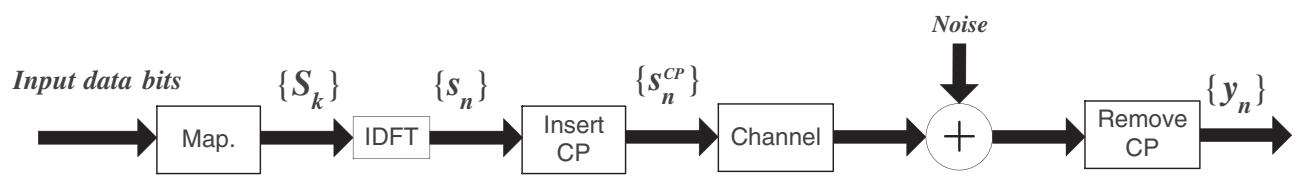

(a)

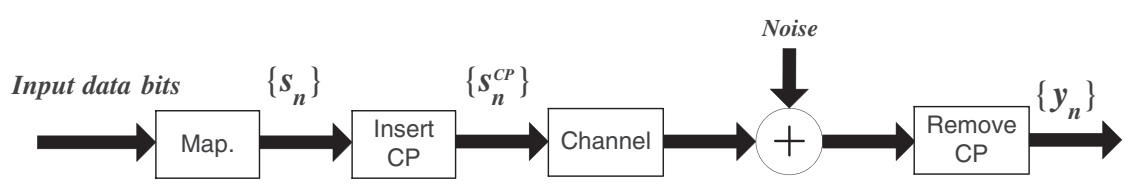

(b)

Figure 1. (a) Orthogonal frequency division multiplexing transmission chain; (b) Single-carrier with frequency-domain equalisation transmission chain.

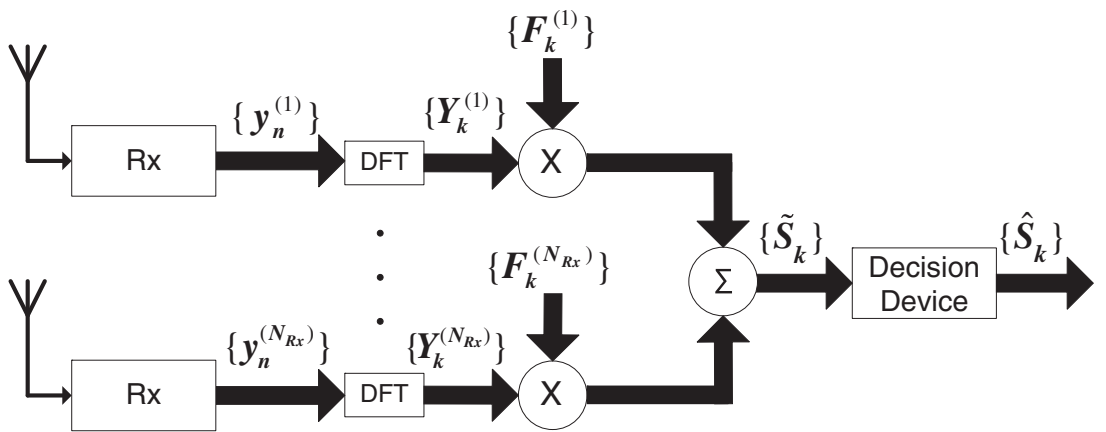

Figure 2. Orthogonal frequency division multiplexing receiver structure with an $N_{R x}$-branch space diversity.

$F_{k}$ that maximise the overall instantaneous signal-to-noise ratio (SNR), associated to the samples $\tilde{S}_{k}$, are [8]

$$
B_{k}^{(i)}=\rho\left(\sum_{l=1}^{N_{R x}} F_{k}^{(l, i)} H_{k}^{(l)}-1\right)
$$

and

$$
F_{k}^{(l, i)}=\frac{\kappa H_{k}^{(l) *}}{\alpha+\left(1-\left(\rho^{(i-1)}\right)^{2}\right) \sum_{l^{\prime}=1}^{N_{R x}}\left|H_{k}^{\left(l^{\prime}\right)}\right|^{2}}
$$

respectively, where $\rho$ denotes the so called correlation factor, $\alpha=E\left[\left|N_{k}^{(l)}\right|^{2}\right] / E\left[\left|S_{k}\right|^{2}\right]$ (which is common to all data blocks and diversity branches) and $\kappa$ selected to guarantee that

$$
\frac{1}{N} \sum_{k=0}^{N-1} \sum_{l=1}^{N_{R x}} F_{k}^{(l, i)} H_{k}^{(l)}=1
$$

As we can see from Equations (6) and (7), the correlation factor $\rho^{(i-1)}$ is a key parameter for the good performance of IB-DFE: Iterative Block Decision Feedback Equaliser receivers because it gives a blockwise reliability measure of the estimates employed in the feedback loop (associated to the previous iteration), which reduces error propagation problems. The correlation factor $\rho^{(i-1)}$ is defined as

$$
\rho^{(i-1)}=\frac{E\left[\hat{s}_{n}^{(i-1)} s_{n}^{*}\right]}{E\left[\left|s_{n}\right|^{2}\right]}=\frac{E\left[\hat{S}_{k}^{(i-1)} S_{k}^{*}\right]}{E\left[\left|S_{k}\right|^{2}\right]}
$$

where the block $\left\{\hat{s}_{n}^{(i-1)} ; n=0,1, \ldots, N-1\right\}$ denotes the data estimates associated to the previous iteration, that is the hard decisions associated to the time-domain block at the output of the FDE, $\left\{\tilde{s}_{n}^{(i)} ; n=0,1, \ldots, N-1\right\}=$ IDFT $\left\{\tilde{S}_{k}^{(i)} ; k=0,1, \ldots, N-1\right\}$.

It should be noted that Equation (5) can be written as

$$
\tilde{S}_{k}^{(i)}=\sum_{l=1}^{N_{R x}} F_{k}^{(l, i)} Y_{k}^{(l)}-B_{k}^{\prime(i)} \bar{S}_{k, \text { Block }}^{(i-1)}
$$

where $B_{k}^{(i)}=B_{k}^{(i)} / \rho^{(i-1)}$ and $\bar{S}_{k, \text { Block }}^{(i-1)}=\rho^{(i-1)} \hat{S}_{k}^{(i-1)}$ (as stated before, $\rho^{(i-1)}$ can be considered as the blockwise reliability of the estimates $\left\{\hat{S}_{k}^{(i-1)} ; k=0,1, \ldots\right.$, $N-1\})$.

For the IB-DFE: Iterative Block Decision Feedback Equaliser performance to be improved, it is possible 